\title{
EXISTENCE OF MAXIMAL IDEALS IN ALGEBRAS OF CONTINUOUS FUNCTIONS
}

\author{
HENRY HELSON AND FRANK QUIGLEY
}

This note is a contribution to the study of the subalgebras of the space $C(S)$ of complex-valued continuous functions on a compact Hausdorff space $S$. We are interested here in finding conditions under which an algebra has maximal ideals other than the obvious ones corresponding to the points of $S$. We shall restrict ourselves to the case where $S$ is a circle or an interval and shall give two sets of hypotheses under which other maximal ideals do exist.

Both theorems depend on deep results about algebras of functions. The first is really a corollary of the theorem of Mergelyan and others which states that on any compact set $E$ in the plane, of plane measure zero, an arbitrary continuous function can be uniformly approximated by rational functions having their poles outside $E$. We are presenting Theorem 1 mainly because its corollary is a statement about polynomials in several complex variables which seems to be new, and which we think is curious.

Theorem 2 depends less obviously on the theorem of Silov asserting the existence of idempotents corresponding to the open-closed subsets of the structure space of a commutative Banach algebra. Our theorem is of Stone-Weierstrass type; it states, under hypotheses, that a given algebra either has many maximal ideals or else contains all continuous functions.

Let $\mathfrak{A}$ be a closed subalgebra of $C(S)$, and let $\mathfrak{M}$ be the collection of functions in $\mathfrak{A}$ which vanish at a given point of $S$. Either $\mathfrak{M}$ is all of $\mathfrak{A}$, or $\mathfrak{M}$ is a maximal ideal in $\mathfrak{A}$, in which case we say that $\mathfrak{M}$ is associated with the given point. If $\mathfrak{M}$ is a maximal ideal which is not of this form, we say that it is not associated with any point of $S$. We can now state our first theorem.

THEOREM 1. Let $S$ be a circle or a closed interval, and let $\mathfrak{A}$ be a proper closed subalgebra of $C(S)$ which contains scalars and separates points. Suppose $\mathfrak{A}$ contains a subset which separates points, and which has the property that each of its functions maps $S$ onto a plane set of Lebesgue measure zero. Then $\mathfrak{A}$ has maximal ideals not associated with points of $S$.

Suppose, on the contrary, that all the maximal ideals of $\mathfrak{A}$ are associated with points of $S$. Then, if $\phi$ belongs to $\mathfrak{A}$ and does not vanish on $S, \phi$ has an inverse in $\mathfrak{A}$. More generally, if $\phi$ is in $\mathfrak{A}$, and

Received by the editors February 28, 1956. 
$R$ is a rational function such that $R(\phi)$ is continuous, then $R(\phi)$ is in $\mathfrak{A}$. Suppose $\phi$ is one of the functions in $\mathfrak{A}$ which map $S$ onto a plane set of measure zero, and denote the image of $S$ under $\phi$ by $E$. It is known [2] that every continuous function on $E$ can be approximated uniformly by rational functions with poles outside $E$. Hence for any continuous function $f$ defined on $E, f(\phi)$ can be uniformly approximated by functions $R(\phi)$ belonging to $\mathfrak{A}$, and so $f(\phi)$ itself belongs to $\mathfrak{A}$. Now by hypothesis we can separate any pair of distinct points $p$ and $q$ in $S$ by a function $\phi$ whose image is a null-set; and $\phi(p)$ and $\phi(q)$ can be separated by a real continuous function $f$. Thus the real functions $f(\phi)$ in $\mathfrak{A}$ separate points on $S$, and $\mathfrak{A}$ must contain all continuous functions by the Stone-Weierstrass Theorem.

Corollary. Let $\Gamma$ be a differentiable arc or closed curve in the space of $n$ complex variables. Either every continuous function on $\Gamma$ can be approximated uniformly by polynomials in $n$ complex variables, or there is a point $q$ in the space outside $\Gamma$ such that $|P(q)| \leqq \sup _{u \in \Gamma}|P(u)|$ for every polynomial $P$.

Indeed, we can take for $\mathfrak{A}$ the continuous functions on $\Gamma$ which are the uniform limits of polynomials. Clearly every polynomial maps $\Gamma$ onto a compact set of measure zero in the plane, and the polynomials separate points on $\Gamma$ as well. So the theorem applies, and either $\mathfrak{A}$ coincides with $C(\Gamma)$, or $\mathfrak{A}$ has maximal ideals not associated with points of $\Gamma$. Let the coordinates of the space be $z_{1}, \cdots, z_{n}$; the $z_{j}$ are elements of $\mathfrak{A}$ when considered as functions on $\Gamma$. A homomorphism of $\mathfrak{A}$ (determined by a maximal ideal) carries the $z_{j}$ into complex constants $q_{j}$; and since the $z_{j}$ are a set of generators for $\mathfrak{A}$, the homomorphism carries an arbitrary function $\phi$ in $\mathfrak{A}$ into the number $\phi\left(q_{1}, \cdots, q_{n}\right)$. Consequently, if the maximal ideal is not associated with a point of $\Gamma$, then $q=\left(q_{1}, \cdots, q_{n}\right)$ does not lie on $\Gamma$. From the general principles of Banach algebras we know that $\left|\phi\left(q_{1}, \cdots, q_{n}\right)\right|$ $\leqq\|\phi\|=\sup _{u \in \mathrm{r}}|\phi(u)|$, and this is what we had to prove.

Theorem 1 and its corollary are very primitive; we have no idea what the situation is when the functions in $\mathfrak{A}$ are all space-filling curves. It is quite possible that the corollary remains true without any hypotheses on the smoothness of the embedding.

TheOREM 2. Let $S$ be a circle or a closed interval, and let $\mathfrak{A}$ be a proper closed subalgebra of $C(S)$ which contains scalars and separates points. Assume moreover that $\mathfrak{A}$ contains a function $e^{i \phi}$, where $\phi$ assumes real

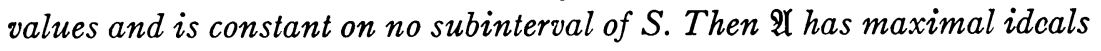
which are not associated with points of $S$. 
If we make the special assumption that $\phi$ is an increasing function, then (as Dr. J. Wermer has pointed out to us) the theorem is a corollary of the generalized Stone-Weierstrass Theorem of Silov [3]. Indeed, $e^{i \phi}$ assumes a given value on a certain finite set of points of $S$, and for each such finite set there is a function of $\mathfrak{A}$ assuming prescribed values at each point. (Since $\mathfrak{A}$ separates points, it is not difficult to construct such a function in $\mathfrak{A}$.) If $\mathfrak{A}$ had no maximal ideals except those associated with points of $S$, then $\mathfrak{A}$ would contain $f\left(e^{i \phi}\right)$ for every continuous function $f$ defined on the circle. Silov's theorem implies then that $\mathfrak{A}$ contains all continuous functions on $S$, contrary to hypothesis. In fact, by an additional argument we can prove that one of the maximal ideals of $\mathfrak{A}$ contains $e^{i \phi}$ itself. All this generalizes part of a theorem of Wermer [5], which characterizes the algebras $\mathfrak{A}$ on the circle which are generated by $e^{2 i x}$ and one other function. Wermer shows that the functions of $\mathfrak{A}$ can be extended to the points of a Riemann surface bounded by $S$, and he describes the surface. The maximal ideals of $\mathfrak{A}$ are the points of $S$ and the Riemann surface. We can assert without any condition of finite generation, and with $e^{n i x}(n=2,3, \cdots)$ in place of $e^{2 i x}$, that $\mathfrak{A}$ has maximal ideals in addition to the points of $S$. We do not know what conditions are necessary in order that the space of maximal ideals should be a Riemann surface.

The situation is less simple if $e^{i \phi}$ assumes a constant value on an infinite set. We shall prove Theorem 2 by contradiction, assuming that $\mathfrak{A}$ has no maximal ideals except those associated with points of $S$. Then $e^{i \phi}$ belongs to no maximal ideal, and so $e^{i n \phi}$ belongs to $\mathfrak{A}$ for all integers $n$. Therefore any finite sum of the form $\sum a_{n} e^{i n \phi}$ belongs to $\mathfrak{A}$; since any continuous periodic function is the uniform limit of trigonometric polynomials, $g\left(e^{i \phi}\right)$ belongs to $\mathfrak{A}$ if $g$ is continuous on the circle.

Let $\mu$ be an arbitrary Borel measure on $S$ whose integral with every function of $\mathfrak{A}$ vanishes. Denote by $\chi_{I}$ the characteristic function of a closed arc $I$ on the circle, and by $E_{I}$ the set of points in $S$ which $e^{i \phi}$ maps into $I$. Let $f_{n}$ be a sequence of continuous functions on the circle decreasing to $\chi_{I}$. Using the fact that $f_{n}\left(e^{i \phi}\right)$ belongs to $\mathfrak{A}$ for each $n$, we have for any $\psi$ in $\mathfrak{A}$

$$
\int_{E_{I}} \psi d \mu=\int \psi \chi_{I}\left(e^{i \phi}\right) d \mu=\lim \int \psi f_{n}\left(e^{i \phi}\right) d \mu=0 .
$$

That is to say, whenever $\mu$ is orthogonal to all the functions of $\mathfrak{A}$, the same is true of that part of $\mu$ concentrated on $E_{I}$.

Now form the algebra $\mathfrak{B}$ of functions defined and continuous on 
$E_{I}$, which can be approximated uniformly on $E_{I}$ by functions in $\mathfrak{A}$; $\mathscr{B}$ is a closed subalgebra of $C\left(E_{I}\right)$ which contains scalars and separates points on $E_{I}$. The points of $E_{I}$ determine distinct maximal ideals of $\mathfrak{B}$; we assert that all the maximal ideals are of this type. In fact, let $F$ be a nontrivial homomorphism of $\mathscr{B}$ into the complex numbers. Since the functions of $\mathfrak{B}$ are the limits on $E_{I}$ of functions of $\mathfrak{A}, F$ is defined (and obviously continuous) on a homomorphic image of $\mathfrak{A}$, and is still not the zero homomorphism. Thus $F$ determines a homomorphism of $\mathfrak{A}$ into the complex numbers, which by hypothesis has the form $F[\psi]=\psi(p)(\psi \in \mathfrak{U})$ for some fixed $p$ in $S$. Since $F$ has bound 1 as a functional on $\mathscr{B}$, we have $|F[\psi]|=|\psi(p)|$ $\leqq \sup _{x \in E_{I}}|\psi(x)|$. If $p$ is not in $E_{I}$, find a continuous function $g$ on the circle which vanishes on $I$ but not at $e^{i \phi(p)}$. Then $g\left(e^{i \phi}\right)$ belongs to $\mathfrak{A}$ and vanishes on $E_{I}$ but not at $p$. The last inequality is therefore not true for all $\psi$ in $\mathfrak{A}$. So we conclude that $p$ belongs to $E_{I}$, and that the functional $F$ on $\mathfrak{B}$ is given by $F[\psi]=\psi(p)(\psi \in \mathfrak{B})$. Thus all the maximal ideals of $\mathfrak{B}$ are associated with points of $E_{I}$.

Returning to the measure $\mu$, we know that the restriction of $\mu$ to $E_{I}$ is orthogonal to all the functions of $\mathfrak{A}$. Since these functions are dense in $\mathfrak{B}$, it follows that $\chi_{E_{I}} d \mu$ is orthogonal to the functions of $\mathfrak{B}$. Now we apply a theorem of Silov [4], which asserts that $\mathfrak{B}$ contains the characteristic function of each subset of its structure space which is open and closed. That is, if $D$ is a subset of $E_{I}$ which is open and closed in $E_{I}$, then for any function $\psi$ of $\mathfrak{B}$ we have $\int_{D} \psi d \mu=0$. The same equation holds a fortiori if $\psi$ is defined on all of $S$ and belongs to $\mathfrak{A}$. So the restriction of $\mu$ to any open-closed subset of $E_{I}$ is orthogonal to all the functions of $\mathfrak{A}$.

Let $p$ and $q$ be distinct points of $S$. We shall find a real function in $\mathfrak{A}$ taking different values at the two points. If $\phi(p)-\phi(q)$ is not a multiple of $2 \pi$, then there is a real function $f$ on the circle such that $f\left(e^{i \phi}\right)$ separates $p$ and $q$; otherwise, at least $\phi$ is not constant on the interval between $p$ and $q$. We can find a closed arc $I$ on the circle containing $e^{i \phi(p)}$ in its interior such that $p$ and $q$ do not lie in a connected subset of $E_{I}$. Let $f$ be continuous on the circle, vanishing outside $I$, but not at $e^{i \phi(p)}$. Then $f\left(e^{i \phi}\right)$ belongs to $\mathfrak{A}$, and vanishes outside $E_{I}$. Let $D$ be an open-closed subset of $E_{I}$ containing $p$ but not $q$. Then $\chi_{D} f\left(e^{i \phi}\right)$ is continuous and separates $p$ and $q$; moreover, for each measure $\mu$ orthogonal to $\mathfrak{A}$ it is clear that $\int_{D} f\left(e^{i \phi}\right) d \mu=0$, by what we have already proved. So $\chi_{D} f\left(e^{i \phi}\right)$ belongs to $\mathfrak{A}$, and separates $p$ and $q$ as required.

Since the real functions of $\mathfrak{A}$ separate points on $S$, the StoneWeierstrass Theorem asserts that every continuous function belongs 
to $\mathfrak{A}$. This is contrary to the assumption that $\mathfrak{A}$ is a proper subalgebra of $C(S)$; therefore the maximal ideals of $\mathfrak{A}$ cannot all be associated with points of $S$, and the theorem is proved.

As a corollary, we observe that if $\mathfrak{A}$ is an integral domain and a maximal subalgebra of $C(S)$, where $S$ is a circle or an interval, and if $\mathfrak{A}$ contains a nonconstant function of modulus one, then $\mathfrak{A}$ has maximal ideals which are not associated with points of $S$. For we have proved [1] that any function of $\mathfrak{A}$ which is constant on an interval is everywhere constant, so Theorem 2 applies.

\section{REFERENCES}

1. H. Helson and F. Quigley, Maximal algebras of continuous functions, Proc. Amer. Math. Soc. vol. 8 (1957) pp. 111-114.

2. S. N. Mergelyan, Uniform approximations to functions of a complex variable, American Mathematical Society Translations, no. 101.

3. G. E. Silov, On rings of functions with uniform convergence, Ukrain. Mat. Zurnal vol. 3 (1951) pp. 404-411.

4. - On decomposition of a commutative normed ring in a direct sum of ideals, Math. Sbornik N.S. vol. 32 (1953) pp. 353-364.

5. J. Wermer, Algebras with two generators, Amer. J. Math. vol. 76 (1954) pp. 853859.

YALE UNIVERSITY 